\title{
474964 - IMPACT OF PREOPERATIVE CHANGE IN PHYSICAL FUNCTION ON SURGICAL RECOVERY: ARGUMENT SUPPORTING PREHABILITATION FOR COLORECTAL SURGERY
}

Francesco Carli, $\mathrm{MD}^{1}$, Patrick Charlebois, $\mathrm{MD}^{2}$, Liane Feldman, $\mathrm{MD}^{2}$, Barry Stein, $\mathbf{M D}^{2}$, Gerald Zavorsky, MSc ${ }^{1}$, Gerald Fried, $\mathbf{M D}^{2}$, Nancy Mayo, $\mathbf{P h D}^{3}$

1. Anesthesia, McGill, Montreal, QC, Canada

2. Surgery, McGill, Montreal, QC, Canada

3. Clinical Epidemiology, McGill, Montreal, QC, Canada

Introduction: We hypothesized that improvement in physical conditioning preoperatively ("prehabilitation”)(1) would result in better postoperative recovery. Methods: Adults scheduled for colorectal resection were enrolled in a daily prehabilitation program (3-6 weeks) consisting of either stationary cycling with weight training (complex) or walking with breathing and leg exercises (simple). Primary outcome was functional walking capacity, reflected by the distance (m) covered in six minutes (Six-Minute-Walk-Test: 6MWT). Patient-reported outcomes were also collected. Evaluations were done at entry, pre-surgery and post-surgery (average 9 weeks). At the end of the prehabilitation period, participants were categorized compared to baseline $6 \mathrm{MWT}$ as improved (gain $>20 \mathrm{~m}$.), unchanged $( \pm 20 \mathrm{~m}$.) or deteriorated $(-20 \mathrm{~m}$.). Preop change status was correlated to recovery of baseline 6MWT postop, using multiple linear regression.

Results: 141 subjects enrolled; 22 were not operated, 48 were unable to comply and dropped out, and 71 completed the program. Dropouts reported a significantly higher degree of symptoms of depression. There was a positive relationship between prehabilitation change and postop recovery $(\mathrm{p}<0.001)$. Compared to baseline, postop $6 \mathrm{MWT}$ was reduced by $7.5 \%$ for people who had deteriorated during prehabilitation $(n=21)$, but increased by $7.8 \%$ for those who had improved $(n=23)$. Other predictors of recovery were age (>75 vs. $<50$ years: $-8.6 \%$ ), BMI (>30 vs. $<25:-8.3 \%$ ), complications (Clavien 2-4 vs. 0-1: -8.2\%) and baseline 6MWT(-4.0\%/100m. less walked). There were no differences between the complex or simple programs.

Discussion: Improvement in preop fitness was associated with postoperative recovery. This provides level IIb evidence to support prehabilitation.

References: 1 . Carli F, Zavorsky G. Optimizing functional exercise capacity in the elderly surgical population. Current Opinion in Clinical Nutrition and Metabolic Care 2005; 8: 23-32 